\title{
Principles, technologies, and applications of plasmonic biosensors
}

Cite as: J. Appl. Phys. 129, 111102 (2021); https://doi.org/10.1063/5.0042811

Submitted: 04 January 2021 • Accepted: 03 March 2021 • Published Online: 17 March 2021

Maria Soler and Laura M. Lechuga

\section{COLLECTIONS}

Paper published as part of the special topic on Plasmonics: Enabling Functionalities with Novel Materials
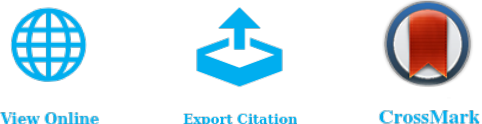

View Online

Export Citation

\section{ARTICLES YOU MAY BE INTERESTED IN}

A plasmonically enhanced route to faster and more energy-efficient phase-change integrated photonic memory and computing devices

Journal of Applied Physics 129, 110902 (2021); https://doi.org/10.1063/5.0042962

Imaging ellipsometry for structured and plasmonic materials

Journal of Applied Physics 129, 113101 (2021); https://doi.org/10.1063/5.0039150

A first-principles understanding of point defects and impurities in GaN

Journal of Applied Physics 129, 111101 (2021); https://doi.org/10.1063/5.0041506

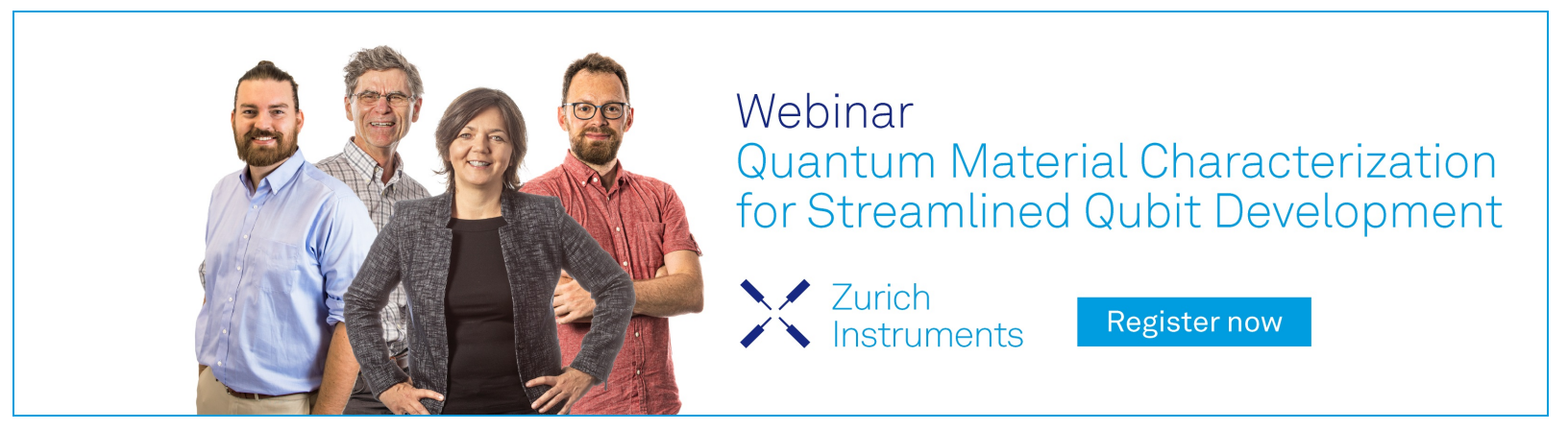




\title{
Principles, technologies, and applications of plasmonic biosensors
}

\author{
Cite as: J. Appl. Phys. 129, 111102 (2021); doi: 10.1063/5.0042811 \\ Submitted: 4 January 2021 . Accepted: 3 March 2021 . \\ Published Online: 17 March 2021
}

Maria Soler ${ }^{\text {a) }}$ (D) and Laura M. Lechuga

\begin{abstract}
AFFILIATIONS
Nanobiosensors and Bioanalytical Applications Group (NanoB2A), Catalan Institute of Nanoscience and Nanotechnology (ICN2), CSIC, BIST and CIBER-BBN, Bellaterra, Barcelona 08193, Spain
\end{abstract}

Note: This paper is part of the Special Topic on Plasmonics: Enabling Functionalities with Novel Materials.

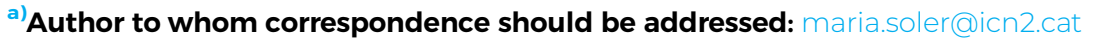

\begin{abstract}
Plasmonic materials and phenomena have been widely studied and applied in multiple fields for a long time. One of the most promising applications is in the engineering of biosensor devices, offering label-free and real-time analysis of biomolecular interactions with excellent performances. In this tutorial, we provide a pedagogical review of the working principles of plasmonic biosensors, main fabrication methods, instrumentation, and general guidelines for their development. Special focus is placed on the biosensor performance characterization and assessment, as well as on the sensor surface biofunctionalization. In the end, we discuss the common procedure to develop and validate biosensors for relevant biomedical and environmental purposes and future perspectives in terms of boosting capabilities and sensor integration in point-of-care platforms.
\end{abstract}

\section{Published under license by AIP Publishing. https://doi.org/10.1063/5.0042811}

\section{INTRODUCTION}

Biosensors have been profiled as the most promising alternative for modernizing biological and chemical analyses, which will have a decisive impact on boosting healthcare and medical assistance-especially in point-of-care diagnosis-as well as in environmental control and monitoring. A biosensor is defined as a self-contained integrated device capable of providing specific quantitative or semi-quantitative analytical information using a biological or biomimetic recognition element, which is in direct spatial contact with a transducer (Fig. 1). The biorecognition layer, typically composed of enzymes, antibodies, or nucleic acids, is designed to specifically interact with the target compound in a sample. When the biochemical interaction occurs, a series of physicochemical changes in the medium or the sensor surface are detected by the transducer and converted into discrete or continuous signals that can be read immediately. The engineering and integration of biosensors into so-called point-of-care (POC) devices offer unique features to improve current analysis techniques in the biomedical or environmental fields. ${ }^{1-4}$ The combination of the bioreceptor layer with the transducer in one single device confers the ability to detect the target analyte with high sensitivity and selectivity in a fast one-step. Moreover, biosensors could ideally overcome important limitations of conventional techniques, such as the need for analyte extraction or purification or the use of additional equipment for signal readout, which is usually operated by specialized personnel. Label-free biosensors, in particular, can also monitor biological interactions in real time, allowing for an evaluation of the affinity and kinetics of such interactions and, thereby, helping to elucidate the biochemical mechanisms involved in a disease or in the evaluation and characterization of drugs, for example. Finally, biosensors also benefit from great versatility, making it possible to evaluate a wide range of analytes just by selecting the appropriate biological receptor. Recent advances in nanotechnology further provide interesting opportunities for biosensor miniaturization, high-throughput, and low-cost production, creating competitive alternatives for point-of-care analysis. ${ }^{5,6}$

The pursuit of obtaining powerful biosensors has experienced exponential growth in the last few decades, encompassing the work of numerous disciplines, such as materials sciences, physics, engineering, molecular biology, chemistry, or biotechnology. The multidisciplinary nature of biosensor research has led to a vast range of biosensor platforms based on different types of biorecognition 


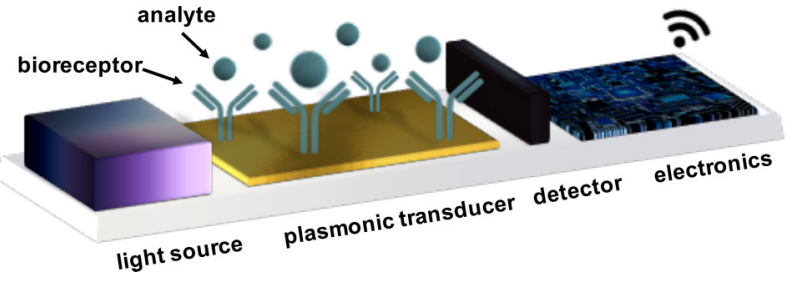

FIG. 1. Illustration of a point-of-care biosensor based on a plasmonic transducer. The integrated device contains a light source and detector, an electronic system for signal readout, and the plasmonic sensor. The sensor surface is functionalized with specific bioreceptors (i.e., antibodies) for the selective interaction with the analyte of interest.

elements (i.e., catalytic or affinity-based) or transducers (i.e., electrochemical, mechanic, optical, etc.). Optical biosensors based on plasmonics are among the most widely studied and employed, with increasing interest in the field, which is continuously introducing novel materials and architectures or demonstrating new high-value applications. ${ }^{4,7}$

In this tutorial, we will provide a review of the most common plasmonic biosensors focusing on the description of the physics and working principles, the instrumentation of the sensing platforms, and the main performance characteristics. We will, as well, provide standard guidelines for proper sensor surface biofunctionalization and a brief comment on the application of plasmonic biosensors to the biomedical and environmental fields.

\section{SURFACE PLASMON RESONANCE (SPR) BIOSENSORS}

Surface plasmon resonance (SPR) systems are founded on an optical phenomenon that was first noticed between 1900 and 1912 by Wood. ${ }^{8,9}$ He observed an unusual dark and light pattern in the reflected light of a metal-backed diffraction grating, and although he hypothesized about light-metal interactions, no clear answer was provided and the phenomenon was termed Wood's anomalies. Along the following years, theoretical studies and analyses were performed by Rayleigh, ${ }^{10}$ Wood, ${ }^{11}$ and Palmer, ${ }^{12,13}$ but it was Fano in 1941 who came to the conclusion that these anomalies were related to surface waves (surface plasmons) supported by the grating structure. ${ }^{14}$ In the 1950 s, further experimental research described the excitation of a surface plasma oscillation of the conducting electrons of the metal, which generates evanescent electromagnetic waves. ${ }^{15-17}$ Soon after, in the late 1960s, the controlled excitation of surface plasmons was achieved by Kretschmann and Raether $^{18}$ and Otto ${ }^{19}$ by means of attenuated total reflection (ATR) using prism-coupler based systems; its first application for sensing was demonstrated by Nylander and Liedberg. ${ }^{20}$ On the other hand, Cullen et al. were first in developing an SPR based on gratingcoupler systems, demonstrating their sensing performance with immunoassays. ${ }^{21}$ Later, in the 1980s, the evanescent field SPR principle also found applications in spectroscopy, as for the interrogation of thin chemical and biological films, which was first demonstrated by Pockrand et al. . $^{22}$

In 1980, the first commercial SPR biosensor was launched (BIAcore. Pharmacia Biosensor AB, Sweden), and ten years later, it was first resold. ${ }^{23,24}$ Since then, the commercialization and use of SPR biosensor systems has widely expanded and numerous manufacturers are selling different platforms with improved capabilities that are routinely employed in research laboratories or the pharmaceutical industry for the analysis of biochemical compounds and interactions. It is worth mentioning that SPR sensors are sold as bare instrumentation, and thus, the application for specific biological or chemical assays has to be developed and optimized by the end user.

\section{A. Physics and working principle}

Surface plasmon resonance refers to the coherent oscillations of charge density that exist at the interface between two media with dielectric constants of opposite signs, such as a metal and a dielectric [Fig. 2(a)]. The surface plasmons are excited by coupling an incident polarized light, and they propagate along the metal- (a)

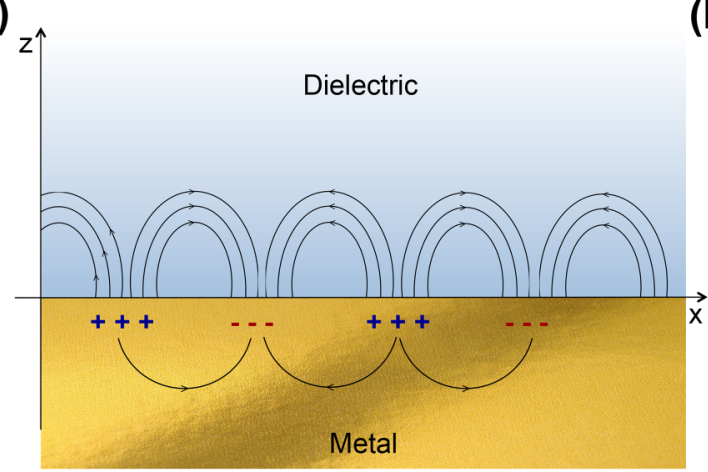

(b)

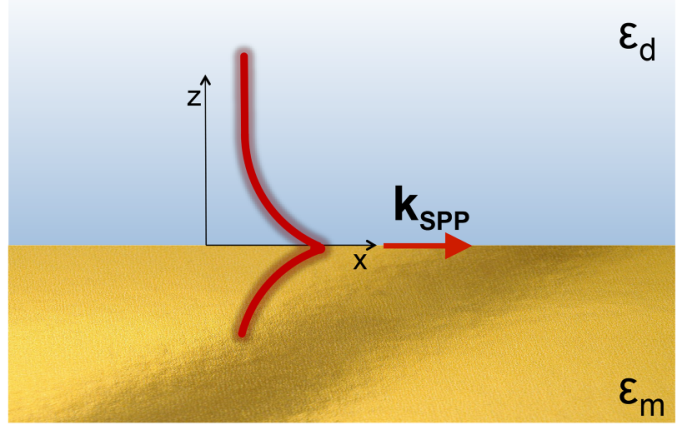

FIG. 2. Schematics of surface plasmon resonance (SPR) at the plane interface of a metal and a dielectric showing: (a) the collective charge oscillation at the surface and (b) the transversal evanescent field distribution. The axes represent the Cartesian coordination system. 
dielectric interface as surface plasmon polaritons (SPPs), behaving like a quasi-free electron plasma and generating an electromagnetic field with an exponentially decaying intensity into both media (i.e., evanescent field). ${ }^{25}$ The SPP is a transverse-magnetic (TM) polarized mode, that is, the magnetic vector is perpendicular to the direction of propagation of the wave and parallel to the plane of the interface. Solving Maxwell's equations with appropriate boundary conditions, the SPP propagation component can be expressed as a function of both the metal and the dielectric permittivity, ${ }^{25-27}$

$$
k_{x}^{S P P}=\frac{\omega}{c} \sqrt{\frac{\varepsilon_{m} \varepsilon_{d}}{\varepsilon_{d}+\varepsilon_{m}}} .
$$

In this equation, $\omega$ is the angular frequency, and $c$ is the speed of the light in vacuum; $\varepsilon_{m}$ represents the frequency-dependent and complex dielectric function of the metal $\left(\varepsilon_{m}=\varepsilon_{m}^{\prime}+i \varepsilon_{m}^{\prime \prime}\right)$ and $\varepsilon_{d}$ is the dielectric constant of the medium, which is directly related to the refractive index $\left(\varepsilon_{d} \approx n_{d}^{2}\right)$. This direct dependency between the propagation vector and the refractive index of the dielectric constitutes the main principle of refractometric sensing platforms.

For the SPP to occur, the equation square-rooted denominator $\left(\varepsilon_{d}+\varepsilon_{m}\right)$ needs to have an absolute positive value; therefore, the real part of $\varepsilon_{m}$ must be negative and its absolute value smaller than $\varepsilon_{d}$. At visible wavelengths, this condition is fulfilled for several metals, from which gold, silver, or aluminum are the most commonly used in plasmonics. Furthermore, due to the relatively small imaginary part of the refractive index of these metals, they show strongly suppressed plasmonic attenuation when compared with other materials, thus minimizing propagation losses. It is worth mentioning here that certain plasmonic behavior has also been observed in non-noble metals and dielectric materials (like graphene), introducing interesting advantages in terms of energy losses or reconfiguration, but their utilization for biosensing has not spread yet. ${ }^{28-30}$ The evanescent field generated by an SPP is confined at the metal-dielectric interface and decreases exponentially into both media [Fig. 2(b)]. The field is distributed in a high asymmetric fashion and most of it is concentrated in the dielectric medium close to the metal surface, showing a typical penetration depth between 50 and $500 \mathrm{~nm}$ when working in visible or nearinfrared (NIR) wavelengths. ${ }^{27,31}$ This is particularly significant for SPR sensing, as it represents the depth probe, meaning that only refractive index changes occurring within the evanescent field penetration depth will alter the SPP propagation and can be detected. Therefore, when a biochemical interaction or biorecognition event takes place at the surface of the plasmonic metal, it can be directly monitored by the interrogation of certain properties of the reflected light, like wavelength, angle, or intensity. The evanescent field-based working principle confers plasmonic sensors with one of the most important assets: the label-free detection capability. This feature enables the direct monitoring of biochemical reactions and quantification of specific analytes without the need for tags (fluorescent or colorimetric), and secondary or amplification steps, therefore simplifying the assay and greatly reducing the analysis turnaround time.

\section{B. Instrumentation}

The different SPR-based optical configurations can be classified on the basis of the SPP excitation method or the detection scheme. The excitation of the SPR is achieved by coupling a light wave to the surface plasmons in such a way that the light's wavevector component parallel to the interface matches the propagation vector of the SPP,

$$
k_{x}^{L i g h t}=\frac{2 \pi}{\lambda} \sqrt{\varepsilon_{d}} \sin \theta=k_{x}^{S P P} .
$$

Generally, the SPP propagation vector is considerably larger than the wavenumber of the light wave in the dielectric; therefore, surface plasmons cannot be excited by direct illumination on smooth surfaces. Common techniques employed for the incoming light coupling make use of prisms, gratings, or waveguides. ${ }^{31}$ Prism couplers are the preferred method for the optical excitation of surface plasmons [Fig. 3(a)]. In the well-known Kretschmann configuration, based on the attenuated total reflection (ATR) phenomenon, light passes through a high refractive index glass prism and it is totally reflected at the prism base, generating an evanescent wave that penetrates the metal film. This evanescent wave propagates along the interface with a certain propagation vector, which can be adjusted to match that of the SPP by controlling the angle of incidence. ${ }^{18}$ Excitation via grating couplers is based on the diffraction of the light waves [Fig. 3(b)]. ${ }^{32}$ The component of the wavevector of the diffracted waves parallel to the interface is increased by an amount inversely proportional to the period of the grating and can be matched to that of the SPP. The excitation of SPP can also be achieved by using optical waveguide structures [Fig. 3(c)]. ${ }^{33}$ The light is guided by a high-refractive index planar optical waveguide-or cylindrical in the case of optical fibers-and, when entering the region with a thin metal layer on top of the waveguide, it evanescently penetrates through the metal exciting an SPP at its outer interface. In the last few years, the use of photonic crystals has emerged as waveguides for SPP excitation and several devices have been realized employing periodic structures, ${ }^{34}$ microstructured fibers, ${ }^{35}$ or Bragg fibers. ${ }^{36}$

Regarding the detection scheme, SPR sensors monitor changes or displacements of the spectral reflectivity dip, which strongly depends on the refractive index of the dielectric. Such interrogation can be based on the angle, intensity, wavelength, or phase.

Angle-based interrogation is one of the most common methods employed in commercial SPR systems. ${ }^{37}$ This configuration uses quasi-monochromatic light sources to excite the surface plasmons, and the SPR curve is obtained by representing the reflectivity as a function of the angle of incidence. Changes in the SPP can be observed by monitoring the entire curve displacements and the minimum value of the SPR dip or by applying the centroid method, which calculates the center of mass of the spectral peak so that it minimizes possible interferences and background noise.

For intensity-based interrogation, both the light wavelength and the incidence angle are fixed. The angle is usually set at the point of maximum slope of the resonance dip. Therefore, changes in the SPP are detected as an increase or decrease of the reflectivity values. This approach has shown interesting advantages, especially 
(a)

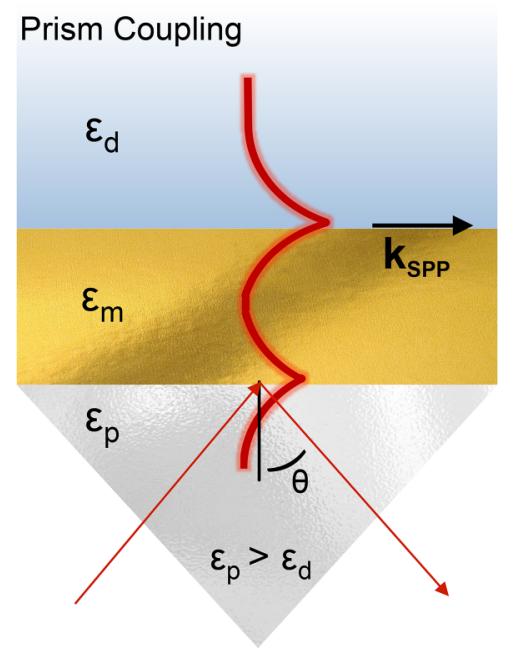

(b)

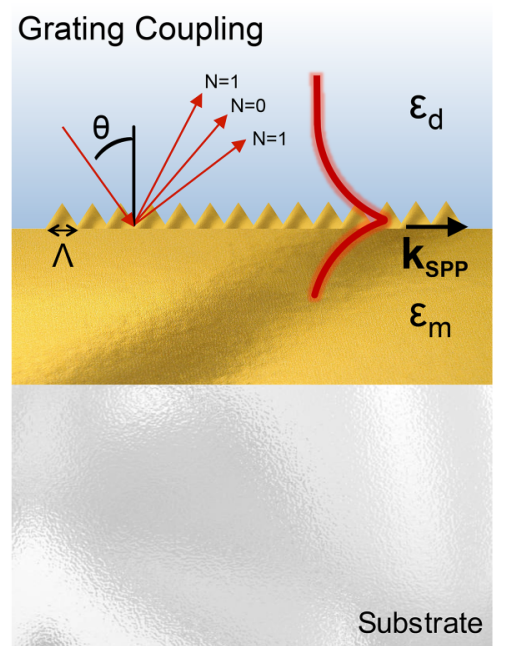

(c)

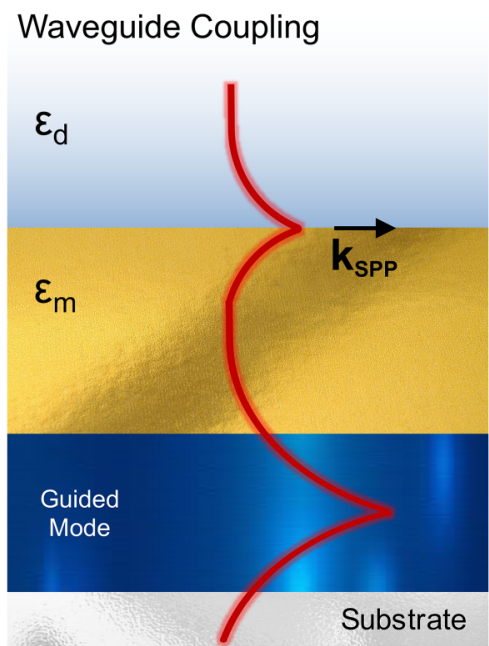

FIG. 3. Schematics of most conventional SPR coupling methods, including (a) prism-coupled Kretschmann configuration, (b) grating coupling, and (c) waveguide coupling. The thick red curves represent the distribution of EM evanescent fields. The red arrows represent incoming light and outputs.

for the development of SPR imaging (SPRi) systems. ${ }^{38}$ By acquiring the reflectivity with two-dimensional detectors, such as chargecoupled devices (CCDs) or complementary metal-oxide sensors (CMOSs), it is relatively easy to extend the label-free and real-time analysis to a multiplexed $2 \mathrm{D}$ array format. With regard to the detector type (CCDs vs CMOSs), much debate still persists in the research community. On the one hand, CCDs have shown higher performances (e.g., sensitivity) and versatility (e.g., customization), especially when working in the near-infrared region. On the other hand, CMOSs outperform CCDs in the visible range, have shown a higher integration potential, and are often more suitable for highthroughput applications. Nevertheless, the implementation of both technologies as SPR detectors can greatly enhance the multiplexing capabilities and boost the miniaturization and integration of the instrumentation. Furthermore, since both wavelength and angle are fixed, this detection scheme avoids the possible noise background produced by mechanical rotors and it allows for using low-cost sources like laser or light emitting diodes (LEDs).

In the wavelength interrogation scheme, a broadband or polychromatic light source is employed for excitation and the SPR curve is obtained at the optimal fixed angle. The reflected light is usually coupled to a spectrometer, enabling the direct interrogation of the plasmonic dip wavelength position. This format provides a high sensitivity and it also allows the use of low-cost sources like halogen lights or LEDs, but the need for high-resolution spectrometers limits the miniaturization and integration capabilities. ${ }^{39}$

In recent years, phase-based interrogation methods have received great attention. This technique evaluates the variation in the phase of the reflected light, which is known to change more abruptly than its intensity. However, measuring light high-frequency oscillations (around $10^{14} \mathrm{~Hz}$ ) requires complex optical readout schemes.
The most common approach employs a polarizer in a configuration similar to ellipsometry. SPR excitation is associated with changes in the p-polarized light, while the s-polarized light remains invariant; therefore, one can extract phase information from the interference of s- and p-polarized light. Other methodologies are based on heterodyne detection, shear interferometry, or spatiotemporal phase modulated interferometry. ${ }^{40,41}$

The latest research in SPR biosensors has been driven by the necessity of offering portable systems able to be deployed at the point of care or in resource-constrained settings. In this regard, the emergence of smartphones with high-performance cameras, LED flashlights, Internet connectivity, and high-resolution screens offers a unique opportunity to incorporate optical biosensors in our daily-use devices. ${ }^{42}$ The CMOS camera of the smartphones can record not only the color (RGB or HVS model) but also the light spectrum, so they can be used for interrogating the SPR transducer signal. Some smartphone-based SPR biosensors have been developed following this scheme; for example, an SPR imaging platform has been developed based on a grating-coupled format using metalcoated Blu-ray disks as sensors. The LED flashlight is used to excite the surface plasmons and the reflected light is passed through a compact disk to spatially disperse the wavelength spectrum, which is imaged using the CMOS camera of the smartphone. A built-in lens on the front of the CMOS allows the image to be focused by touching the screen and the zoom-in of the camera enables magnification. ${ }^{43}$ An angle-resolved prism-coupled SPR detection system has also been demonstrated using a disposable cartridge containing the gold sensor and assembled on the front camera for detection. The device uses the smartphone light source, and the spectral dip of the reflected light is traced on the screen as a function of the angle and wavelength. ${ }^{44}$ This demonstrates that, although being a 
relatively mature technology, research on SPR biosensors is still ongoing actively and, in combination with other cutting-edge technologies, it might soon become a powerful diagnostic tool within the reach of everyone.

\section{NANOPLASMONIC BIOSENSORS}

Amid the rise of nanotechnology, research on plasmonic biosensing has discovered and incorporated new features and phenomena, only occurring at subwavelength dimensions and able to improve the resolution, performance, and integration capabilities of the sensor systems. Although nanoplasmonics is considered a relatively recent research field, the first uses of the exceptional effects of light-matter interaction at the nanoscale date back to the 4th century, with the Lycurgus Cup being the best example, an ancient Roman cage-cup currently exposed in the British Museum. ${ }^{45}$ This vessel is made of dichroic glass containing silver nanoparticles. The cup appears green when viewed in the reflected light, whereas when light is transmitted from the inside, it appears to be red, illustrating the plasmonic phenomena of nanoparticles. However, controlled fabrication and characterization of nanoparticles was not achieved until a few decades ago. With the implementation of nanofabrication techniques like e-beam lithography (EBL) or focused ion beam (FIB) lithography, high-precision nanostructured surfaces began to be evaluated as sensor transducers. These nanostructures show interesting advantages compared with propagating SPR, such as the excitation of plasmonic resonance by direct illumination, overcoming the need for complex light coupling systems, or the enhanced near-field evanescent field, which might increase the sensor surface sensitivity. Today, a few nanoplasmonic biosensors have arrived in the market (e.g., Nicoya in Canada, or LSPR AG in Switzerland), but their implementation as routine testing instrumentation in laboratories or clinics has not been yet accomplished. Research on nanoplasmonics is still on the way to incorporate all assets provided by the nanotechnology, making a truly operative and robust system that can beat the conventional SPR biosensor.

\section{A. Physics and working principle}

Light interaction with subwavelength-sized metallic nanoparticles arises in the so-called localized surface plasmon resonance (LSPR), a non-propagating oscillation of conducting electrons. ${ }^{46,47}$ This effect is due to the accumulation of polarization charges on the surface of the nanoparticle, which is acting as a dipole (Fig. 4). The dipolar field is responsible for the enhanced absorption and scattering of the light, as well as for the strongly enhanced EM field in the close vicinity of the nanoparticle surface. ${ }^{48}$

To simplify the theoretical description of the LSPR principle, we can consider a metallic spherical nanoparticle with $\varnothing<<\lambda$, where $\varnothing$ is the diameter of the particle and $\lambda$ is the wavelength of an incident light. Under this condition, the external EM field appears static around the nanoparticle and the charge oscillation behaves as a single dipole with amplitude that is strongly influenced by the distance between the surface charges. ${ }^{46,47}$ Herein, the LSPR condition is related to the polarizability $\left(\alpha_{0}\right)$ of the particle, which is given by

$$
\alpha_{0}=4 \pi^{3} \frac{\varepsilon_{m}(\lambda)-\varepsilon_{d}}{\varepsilon_{m}(\lambda)+2 \varepsilon_{d}}
$$

The polarizability represents the distortion of the electron cloud in response to the external EM field and basically depends on the size of the particle $(\varnothing)$, and the dielectric functions of the metal $\left(\varepsilon_{m}=\varepsilon_{m}^{\prime}+\mathrm{i} \varepsilon_{m}^{\prime \prime}\right)$ and the surrounding medium $\left(\varepsilon_{d} \approx n_{d}^{2}\right)$. The maximum polarizability is achieved when the absolute value of the denominator approaches zero; hence, the LSPR is observed when $\varepsilon_{m}^{\prime}=-2 \varepsilon_{d}$. For noble metals, the dielectric function $\left(\varepsilon_{m}^{\prime}\right)$ shows a strong dependency on the electromagnetic wavelength; therefore, the bright colors exhibited by nanoparticles strictly rely on the exact wavelength at which the LSPR resonance condition is satisfied. For the two most used plasmonic materials, gold and silver, this condition is satisfied in the visible region of the light spectrum, at $560 \mathrm{~nm}$ for $\mathrm{Au}$ and $450 \mathrm{~nm}$ for $\mathrm{Ag}$, respectively.

This theory can be extended to larger or nonspherical nanoparticles, revealing the appearance of LSPR modes with higher multipoles, where half of the electron cloud moves parallel and half (a)

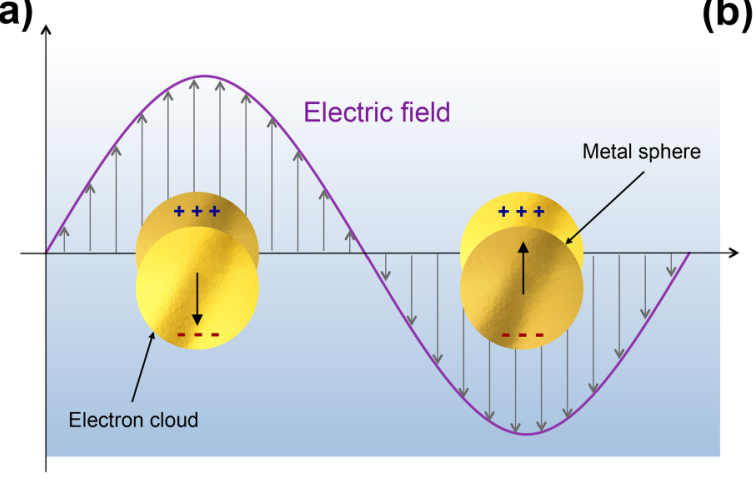

(b)

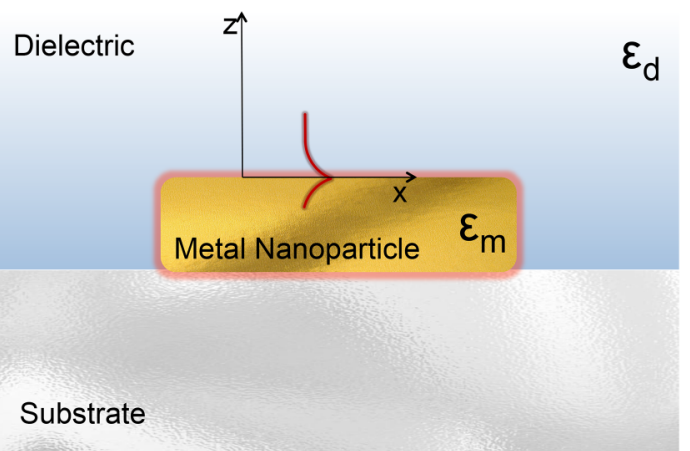

FIG. 4. Schematic representation of (a) LSPR of spherical nanoparticles positioned in a static electric field and (b) the evanescent field distribution of a metal nanostructured surface. The axes represent the Cartesian coordination system. 
anti-parallel to the external EM field. Particularly for ellipsoidal nanoparticles, such as nanorods, surface plasmons split into two distinct modes due to surface curvature and symmetry exhibiting strong polarization-dependent spectra, where small changes in aspect ratio result in significant changes in the absorption band. This size and shape-dependency leads to another important property of plasmonic nanostructures: the spectral tunability. The morphology, size, and distance separation between the nanostructures contribute to the spectral signature of its resonance, dictating the bandwidth and peak position of the LSPR. By varying the size and shape of the plasmonic nanostructures, the LSPR can be tailored and tuned along the entire VIS and NIR regions of the spectrum, so it can fit the most suitable wavelength for specific applications. ${ }^{49}$ On the other hand, extensive research effort has been put into shaping the optical spectra of metallic nanostructures by playing with the constructive and destructive interferences of the plasmon modes in order to achieve the narrowest bands. In particular, Fano-type interferences have demonstrated a significant reduction of the spectral linewidth of plasmonic resonances. ${ }^{50}$ Such narrow bands can be achieved by sculpting multi-particle assemblies (e.g., dimers, trimers, or oligomers) and metasurfaces that exploit the nanometer gaps between plasmonic resonators as dark modes. The unphased coupling of the two modes (bright and dark modes) leads to a narrow resonant band with typical asymmetric line shape, which can enhance the biosensor sensitivity.

Besides the material, size, and shape, the LSPR strongly depends on the dielectric constant of the medium surrounding the nanostructures. Changes in the RI of the medium within the evanescent field lead to changes in the polarizability, which results in displacements of the LSPR peak. In contrast to propagating SPR, the LSPR evanescent field is strongly confined to the particle surface exhibiting a rapid decay in the dielectric medium (typically of a few tens of $\mathrm{nm}$ ). Due to the smaller penetration depth of the evanescent field, the bioreceptors attached to the nanoparticle surface occupy a much larger fraction of the field, which can confer to LSPR sensing high-resolution detection, even at the level of single particle analysis, for instance.

\section{B. Fabrication of plasmonic nanostructures}

The fabrication of nanoplasmonic sensors can be achieved by either top-down or bottom-up methodologies. The former group relies essentially on lithography patterning techniques, while the latter involves depositing chemically synthesized colloidal nanoparticles to a solid support, commonly, glass substrates.

There is an endless variety of colloidal nanoparticles with different geometries, including spheres, rods, triangles, plates, cubes, pyramids, stars, prisms, tubes, etc., which can be fabricated with different synthesis techniques, like seed-mediated growth, anisotropic synthesis, or template-assisted techniques. For a detailed description of colloidal plasmonic nanoparticle fabrication techniques, we refer to comprehensive reviews published elsewhere. ${ }^{51,52}$ Our focus here is directed to the assembly of nanoparticles onto the solid support. Nanoparticles can be attached to previously functionalized glass surfaces via covalent binding or electrostatic interactions. Glass substrates can be modified with either thiol- or amine-functional groups via silanization or other polymeric procedures, which can strongly immobilize gold nanoparticles on the surface by chemisorption (i.e., thiol-gold interaction) or highaffinity interaction (i.e., amine-gold interaction). However, a major problem is nanoparticle aggregation that leads to low reproducible and low efficiency coverage. To solve this, nanoparticles can be initially covered with a functional protective layer (PEGylated compound), carrying carboxyl, amine, thiol, or biotin groups, for example. This step allows for chemically binding the particles to a functionalized substrate, avoiding aggregation and formation of multilayers. The surface density of nanoparticles can be ultimately controlled by optimizing the concentration of colloidal suspension, incubation time or temperature, etc. However, it is not possible to control the nanoparticle distribution over the surface for the formation of ordered periodic arrays.

The fabrication of well-defined arrays of nanostructures can be achieved by top-down fabrication methods, such as photolithography, electron beam lithography (EBL), or focused ion beam lithography (FIB) (Fig. 5). FIB lithography involves directly bombarding the metal sample with gallium ions to draw and sculpt the desired architecture. EBL uses electrons to write a pattern on a sensitive resist layer covering the sample, generally polymethyl methacrylate (PMMA). Then, the substrate is developed and the resist is lifted off, leading to the desired nanostructured metallic surface. Both FIB and EBL methods allow customized and highly precise pattern designs with resolutions of just a few nanometers and are widely used for systematic studies and evaluation of different geometries and architectures of nanoplasmonic sensors. However, both lithographic processes are extremely slow, require high-cost instrumentation, and are limited to patterning only a few $\mu \mathrm{m}^{2}$ areas. Faster, larger scale, and lower cost nanofabrication can be otherwise achieved by following other photolithography approaches, such as nanosphere lithography (NSL) or hole-mask colloidal lithography (HCL). Both methods involve forming a self-assembled layer of nanosphere particles onto the substrate employed as a sacrificial mask for generating the nanostructured surface by subsequent etching and metal deposition steps. The difference between the two
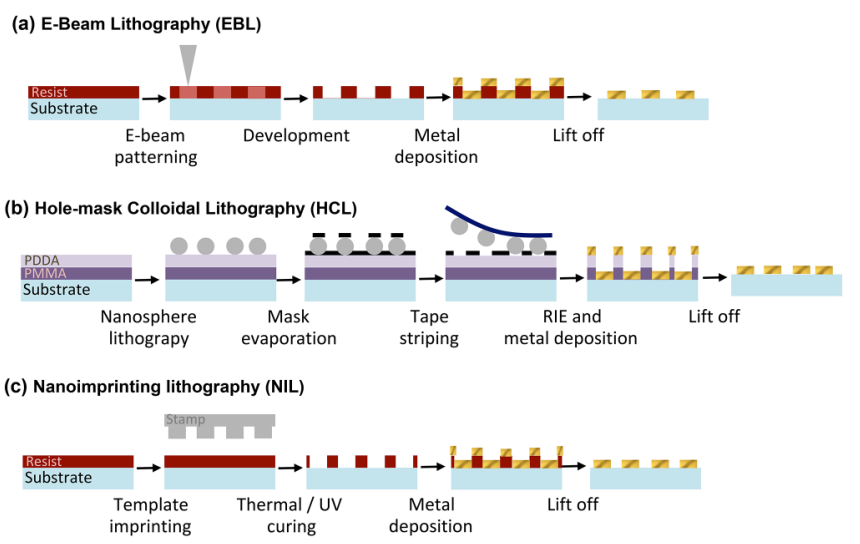

FIG. 5. Schematics of common nanofabrication processes for nanoplasmonic sensors: (a) e-beam lithography, (b) hole-mask colloidal lithography, and (c) nanoimprinting lithography. 
techniques is that NSL renders a highly ordered patterning due to a close-packed layer of nanoparticles, while HCL results in shortordered arrays of nanostructures. Another interesting technique is nanoimprint lithography (NIL). This technique employs a reusable master stamp to transfer predefined patterns to the desired substrate. The master stamp is usually fabricated through EBL, enabling sub-micrometer resolution patterning, and can be reused for more than a thousand times, making NIL a low-cost and scalable technique. Furthermore, it provides a high versatility for fabricating nanostructures of different sizes and shapes, such as disks or dots, holes, or domes. Finally, besides these approaches, other nanofabrication strategies worth mentioning are the nanostencil lithography, ${ }^{53}$ based on shadow-masked patterning of nanostructures, or the interference lithography, ${ }^{54}$ where an interference pattern is written on the photoresist material, which are promising for large-scale and cost-effective production of nanoplasmonic sensors.

\section{Instrumentation}

There are two main categories of nanoplasmonic biosensor technologies: based on nanoparticles (i.e., LSPR biosensors, described in previous sections) and based on nanoapertures. The latter generally consists of arrays of subwavelength apertures fabricated on plasmonic thin films, and its sensing principle relies on the so-called extraordinary optical transmission (EOT), which arises as a combination of both propagating and localized SPR. ${ }^{55,56}$ In both cases, the plasmonic resonance is characterized by the extinction wavelength peak (i.e., LSPR or EOT peak), corresponding to the maximum light absorption and scattering, which can be monitored to detect refractive index changes occurring on the nanostructured surface. One of the major advantages of nanoplasmonic biosensors compared with conventional propagating SPR biosensors is the possibility of exciting the plasmonic resonance by direct illumination, overcoming the need for complex prism- or grating-coupling schemes.
The configuration of nanoplasmonic biosensor platforms generally depends on the nanostructure surface density. For highdensity nanostructured surfaces, extinction measurements are the easiest way to characterize the optical response [Fig. 6(a)]. Light is directly shed on the plasmonic nanostructures and the transmitted light is acquired with either a spectrometer, for wavelength interrogation, or with a camera (CCD or CMOS), for intensity interrogation. A clear example of such configuration is the nanohole-array based sensor. ${ }^{56,57}$ Periodic arrays of gold nanoholes act as grating structures to couple normally incident light and excite the plasmon resonance. The EM field enhancement at the nanoholes enables the EOT, characterized by specific extinction peaks highly sensitive to dielectric refractive index changes. Portable optical readers can be assembled with off-the-shelf optical components, such as LED matching the EOT wavelength (usually in the VIS-NIR range), a microscope objective, and a CMOS sensor. This configuration is the preferred one for portable point-of-care LSPR biosensors, as it eliminates the optical components required for light coupling and allows the use of low-cost sources and detectors. Moreover, extinction measurements offer easy multiplexing and high-throughput capabilities, as large nanoplasmonic surface areas can be illuminated and interrogated simultaneously.

On the other hand, for low-density nanostructured surfaces, a much higher contrast between the incident light and the light absorbed by the nanoparticles is needed. In such cases, scattering measurements offering higher signal-to-noise ratios are the most suitable. Dark-field (DF) microscopy or total internal reflection (TIR) microscopy is usually employed. DF microscopy works in transmission configuration, using a high numerical aperture condenser to focus the nanostructured surface [Fig. 6(b)]. The scattered light dispersed by the nanostructures is collected by a microscope objective with a lower numerical aperture. In TIR microscopy, the LSPR is excited through a prism-coupling scheme, also employing a microscope objective to collect the scattered light, without any restriction on the numerical aperture [Fig. 6(c)]. (a)

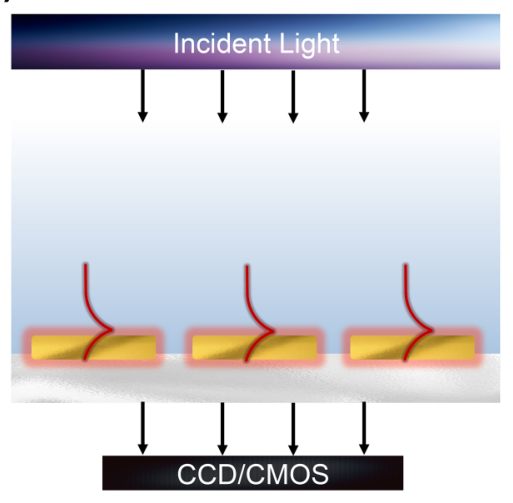

(b)

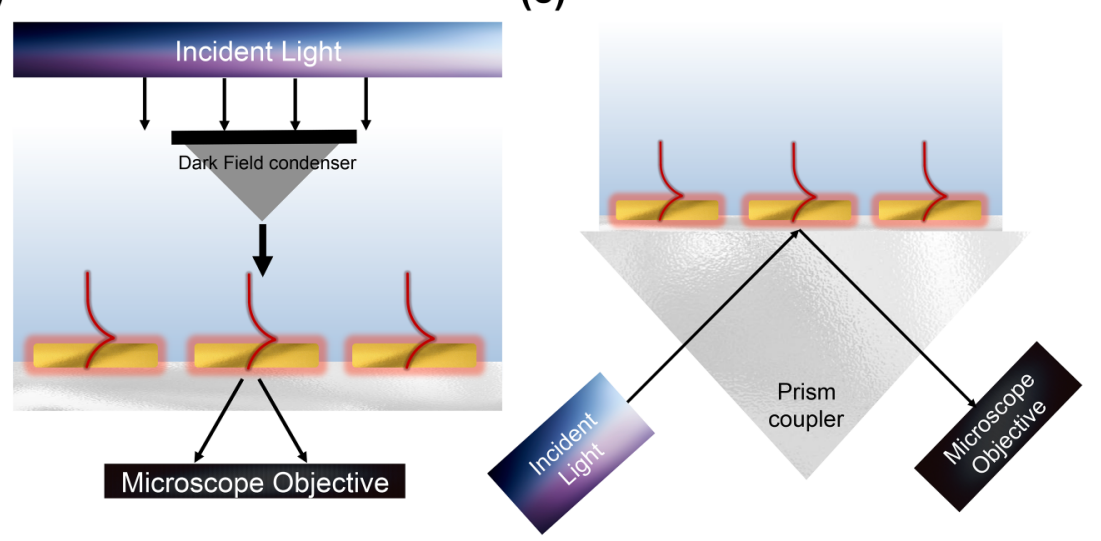

FIG. 6. Diagrams illustrating nanoplasmonic-based biosensor setups: (a) extinction measurements, (b) dark-field microscopy, and (c) total internal reflection (TIR) microscopy. 
Generally, scattering measurements offer a higher control than extinction configuration and permit the evaluation at the single particle level. However, both of them require complex equipment and the integration in portable devices is not straightforward.

Finally, it is worth mentioning that nanoplasmonic sensors can also be employed in traditional Kretschmann-based SPR systems working in wavelength interrogation, just taking into account the different incident light polarization: a transverse-magnetic (TM) mode is required for propagating SPR, while the transverse-electric (TE) mode should be used for LSPR nanostructures. This optical configuration can employ the already optimized instrumentation of advanced SPR biosensors, many of them already in the market, for enhancing certain analytical features (e.g., sensitivity, multiplexing, background noise reduction, etc.) as well as accurately compare the performance of novel plasmonic nanostructures with the standard SPR sensor.

\section{PERFORMANCE OF PLASMONIC BIOSENSORS}

Performance indicators and comparison between different sensor technologies are still subject to controversy in the field of plasmonic biosensors. The idea of developing novel platforms that outperform the conventional ones requires a prior establishment of the performance characteristics to be compared and reported on a benchmark basis, which unfortunately has not been fully established yet. The overall performance of a biosensor should be evaluated not only in terms of sensitivity but also in terms of robustness and reliability of the results (i.e., selectivity, reproducibility, etc.), which are influenced by several factors such as the biological interface, the quality of the sensor structures, or the setup instrumentation. This has increased the need for including numerous performance indicator parameters, and unfortunately, not all of them are determined and reported in published studies.

\section{A. Performance indicators}

The simplest sensitivity parameter in refractometric biosensors is the bulk sensitivity $\left(\mathrm{S}_{\mathrm{B}}\right)$, which quantitates the capability to detect minute changes of the refractive index of the dielectric medium in contact with the sensor surface. The bulk sensitivity is calculated as the sensor response (i.e., $\Delta \lambda, \Delta \theta$, and $\Delta \mathrm{I}$ ) per refractive index variation $(\Delta \mathrm{n})$, expressed in refractive index units (RIUs). To experimentally determine the bulk sensitivity, the sensor is exposed to different liquids (ethanol, acetone, water, etc.) or solutions at different concentrations (glycerol, $\mathrm{HCl}$, etc.) and the signal is plotted vs the RI of the media. The slope of the calibration plot represents the $\mathrm{S}_{\mathrm{B}}$. The smallest detectable refractive index change determines a system's limit of detection (LOD), also commonly referred to as sensor resolution. The sensor resolution is calculated as three times the standard deviation of the baseline noise over the bulk sensitivity. Generally, the resolution of both plasmonic and nanoplasmonic biosensors is found between $10^{-5}$ and $10^{-6}$ RIUs, although some of the more advanced technologies have reached up to $10^{-7}$ RIUs. In terms of bulk sensitivity, however, significant differences can be observed depending on the systems and structures. ${ }^{58,59}$ For example, bulk sensitivity in the simplest plasmonic nanostructures (nanospheres, nanodisks, nanorods, etc.) tends to range between 300 and $500 \mathrm{~nm} / \mathrm{RIU}$, and these values increase for more sophisticated geometries, such as nanoprisms $(600 \mathrm{~nm} / \mathrm{RIU}),{ }^{60}$ ring-disk nanocavities $(650 \mathrm{~nm} / \mathrm{RIU}),{ }^{61}$ or nanocrosses $(1000 \mathrm{~nm} / \mathrm{RIU}){ }^{62}$ Conventional SPR biosensors, in contrast, outperform nanoplasmonics in bulk sensitivity, reaching up to one order of magnitude higher values. ${ }^{63}$ What might seem a discrepancy finds a logic when taking into account the confinement of the evanescent EM field. In propagating SPR, the larger evanescent field penetration depths allow the probing of much larger volumes of the dielectric, thereby increasing the $S_{B}$ values.

From a biosensing point of view, however, the generic bulk sensitivity is not the most appropriate performance indicator. In plasmonic biosensors, the assay is done on the sensor surface, where the evanescent EM field is more intense, and thereby the surface sensitivity $\left(\mathrm{S}_{\mathrm{S}}\right)$ parameter is more useful. $\mathrm{S}_{\mathrm{S}}$ is defined as the sensor response to the surface coverage (i.e., the number of molecules adsorbed on the sensor), and it is often expressed in terms of mass per area (e.g., $\mathrm{pg} / \mathrm{mm}^{2}$ ). To determine the $S_{S}$, different experimental approaches can be used, such as quartz crystal microbalance (QCM) analysis, but it needs to be carried out as additional independent measurements and the results tend to be approximated. The surface sensitivity can also be determined in terms of adlayer thickness, which refers to the sensor response due to the addition of a homogeneous layer of certain material on the surface. It is usually calculated through layer-by-layer deposition of positively and negatively charged polymers, the density and refractive indices of which are well established and available in the literature. The layer thickness of the polymers can be accurately determined by ellipsometry and directly related to the sensor signal. For SPR biosensors, the best surface sensitivity has been reported for the newest Biacore systems $\left(1 \mathrm{pg} / \mathrm{mm}^{2}\right)$. Theoretically, in LSPR biosensors, this value could be largely improved, thanks to the spatial confinement of the EM field at the nanostrucure surface, which ensures a larger occupancy of the probe area by the biomolecular interaction. However, experimentally, only an improvement up to $15 \%-20 \%$ has been demonstrated for some systems. ${ }^{49,64}$ The challenge here is the necessity to assure a homogeneous coverage of the sensor for a good assessment of the surface sensitivity, which is especially complex for more sophisticated nanostructures and architectures.

Besides the different sensitivity indicators, the sensing performance of plasmonic biosensors is strongly influenced by the spectral shape and the background noise of the readout system. Regarding the spectral shape, the most important parameter is the full width half maximum (FWHM), which is the width of the spectral peak (Lorentzian function) measured at half of its amplitude. Reasonably, a narrow spectral band will facilitate the quantitative detection of minimum displacements of the peak position. The FWHM is closely related to the propagation distance of SPR and the plasmon lifetime in LSPR, respectively, and it can be minimized through the rational design and optimization of the plasmonic nanostructure geometry and architecture. For example, structures supporting Fano resonances lead to sharp asymmetric peaks that have shown up to twofold enhanced sensitivity when compared with SPR sensors. ${ }^{65-67}$

The relation between the bulk sensitivity and the FWHM determines the figure of merit (FOM) of the sensor,

$$
F O M=\frac{S_{B}}{F W H M} .
$$


The FOM is the most used parameter for comparing the performance of different biosensor systems. However, it is worth noting that the figure of merit is dependent on the metal film, the prism material, and the resonant wavelength. For instance, the SPR peak of silver structures tends to be sharper (i.e., it falls at a shorter wavelength) than that of the gold ones. But also, at longer wavelengths, the evanescent field has a larger penetration depth, so it is more sensitive to RI changes, and larger shifts of the resonance are expected. All in all, the optimum performance of a biosensor must be judged after taking into account different factors that should be carefully studied, before reaching a compromise between all parameters.

Finally, another interesting sensitivity indicator, especially for bioanalytical applications, is the concentration sensitivity $\left(\mathrm{S}_{\mathrm{C}}\right)$. This value relates the sensor response and the analyte concentration in solution. From this, the analytical limit of detection (LOD) can be determined, as the minimum amount of analyte that can be reliably detected. It is experimentally obtained through interpolation in standard calibration curves, i.e., triplicate assays of serial dilutions of the sample, and calculated as the analyte concentration corresponding to three or five times the standard deviation of the background (when considering a direct assay). It can be expressed in molar (nM, pM), mass per volume $(\mathrm{ng} / \mathrm{mL}, \mathrm{g} / \mathrm{L})$, parts per million or billion (ppm, ppb), etc., depending on the analyte and sample types. Another interesting parameter is the limit of quantification (LOQ), which represents the minimum concentration that can be reliably quantified, usually the starting point of the linear range, and it is calculated as 10 times the standard deviation of the background. Both parameters depend on the bioreceptor quality and affinity, its orientation and density on the sensor surface, and the physicochemical conditions of the assay ( $\mathrm{pH}$, salinity, etc.), hence they cannot be broadly employed when comparing different biosensor platforms. Instead, the analytical sensitivity is often used when developing biosensor applications to evaluate the capability for addressing specific scenarios, for example, the detection of certain levels of clinical biomarkers in blood or monitoring the levels of contaminants in water, and to compare the results with the ones from other analytical techniques like enzyme-linked immunosorbent assay (ELISA) or polymerase chain reaction (PCR) based assays.

\section{B. Strategies for performance improvement}

The most critical aspect for improving the biosensor performance is to increase the signal-to-noise ratio, which has a direct influence on the determination of both the sensor resolution and the analytical sensitivity. The intrinsic background noise of a sensor system can be due to multiple factors, including detrimental substrate effects and the system instrumentation. It has been reported that the underlying substrate of a nanoplasmonic sensor imposes significant drawbacks that affect the sensing performance. Often, the metal nanoparticles are attached to the substrate with aid of a thin metal adhesion layer (e.g., Ti or Cr). The presence of this adhesion metal increases the dephasing time of LSPR, which reduces the scattering amplitude, widening the resonance peak. To overcome this problem, some sensors opt for using a functional chemical matrix (e.g., glass silanization) for the immobilization of the nanoparticles on the substrate, although this approach may induce reproducibility and robustness problems. ${ }^{68}$ But the background noise can also be directly related to the instrumentation, such as light fluctuations, detector resolution, or electronic processing of the signal. Reduction of this noise can be achieved by using high-quality equipment (spectrometers, lasers, etc.) but also with the incorporation of temperature controllers, vibration isolators, and advanced signal postprocessing. Data treatment with algorithms is commonly employed for improving the signal readout, such as using a polynomial fit or the centroid method instead of using the raw spectral data for tracking the peak position. It is also possible to apply electronic filters based on statistical data analysis to hinder and minimize intrinsic signal fluctuations due to the instrumentation.

Substrate effects are also important in the EM field distribution and intensity. Most of the supporting substrates are made of glass, which has a much higher RI than the bioassay sample (i.e., aqueous solutions). This contrast breaks the evanescent EM field symmetry around the nanostructures, which largely shifts toward the metal/glass interface (insensitive to external RI changes), therefore lowering the overall surface sensitivity. The straightforward approach to solve this is to use low refractive index materials as substrate, like Teflon $(\mathrm{n}=1.32)$, which has been shown to improve the bulk sensitivity up to $40 \% .{ }^{69}$ Another strategy involves distancing the plasmonic nanostructures from the surface by placing them on nanopillars or nanopedestals, and, therefore, increasing the particle surface available for binding. This approach has been demonstrated to improve the surface sensitivity for biomolecular assays; however, it requires the introduction of delicate nanofabrication steps, such as the isotropic etch of the glass substrate. ${ }^{70}$

The ultimate strategy for achieving optimum biosensor performance relies on the sensor surface biofunctionalization. The plasmonic transducer surface needs to be modified for attaching the bioreceptor elements that will selectively capture and detect the analyte, but it is also important that the sensor surface can prevent and repeal non-specific adsorptions of non-relevant sample matrix components. ${ }^{71}$ A careful consideration of factors such as the bioreceptor orientation and density, a site-selective immobilization on the resonant hotspots, the final distance between the target biomolecule and the sensor surface, the antifouling properties or the use of specific blocking agents, and even the selection of the bioreceptor itself will be key for enhancing the analytical features of the plasmonic biosensor. This aspect is often under-featured in the development of novel biosensors, but it offers a wide scope for improving the sensing performance; therefore, we feel that it deserves a comprehensive and detailed discussion.

\section{SENSOR SURFACE BIOFUNCTIONALIZATION}

In a label-free plasmonic biosensor, analytical sensitivity and selectivity strongly depend on the biorecognition element tethered to the sensor surface. Typically, in affinity-based biosensors, the most employed biological receptors are antibodies, nucleic acids, or cell membrane receptors, although specifically designed peptides, aptamers, or molecularly imprinted polymers can also be employed. These biomolecules show extraordinary affinity and specificity toward certain analytes, such as the corresponding antigen 
or the complementary oligonucleotide chain, allowing a selective capture of the target compound (analyte) with a high sensitivity. The immobilization procedure onto the sensor surface must include several factors for achieving an optimum biosensor: (i) the packaging density and orientation of the biorecognition element, (ii) the activity and stability during the analysis time, and (iii) the possible interferences or non-specific adsorption of other substances present in the sample matrix.

Sensor biofunctionalization procedures have been developed since long ago, aiming to provide the optimal analytical performance. Physical adsorption (or physisorption) is the simplest strategy to attach the bioreceptor to the sensor surface, which takes advantage of intermolecular forces like electrostatic, hydrophobic, and/or polar interactions [Fig. 7(a)]. Although it is a widely employed procedure in solid-based assays, such as ELISA, physical adsorption suffers from important drawbacks when dealing with (a)

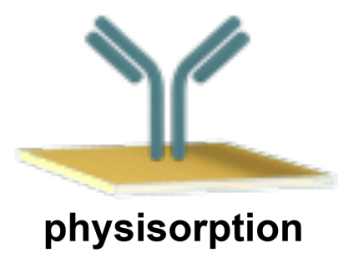

(c)

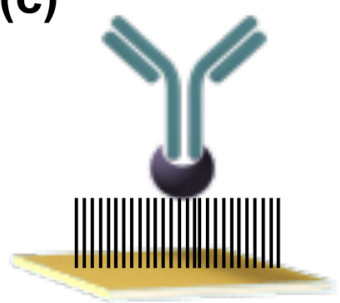

protein A/G

(e)

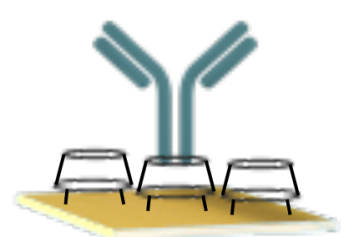

calixarenes (b)

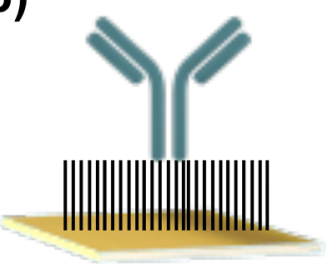

covalent binding

(d)

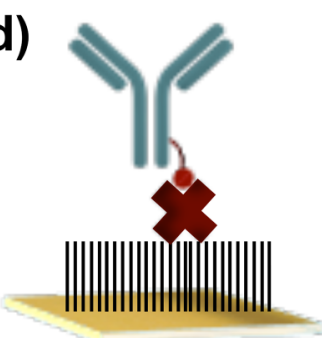

biotin / avidin
FIG. 7. Schematic illustrations of common sensor biofunctionalization strategies: (a) direct physical adsorption, (b) covalent binding to a self-assembled monolayer, (c) via protein A or $G$, (d) via biotin/avidin pair, (e) calixarene-mediated, or (f) via DNA-hybridization. biosensors. Flow-through assays or changes in the $\mathrm{pH}$ or buffer composition can lead to desorption of the biomolecules. Moreover, the uncontrolled interaction of the biomolecules with metallic surfaces can cause denaturation, unfolding, or loss of affinity for the analyte. This is especially important for protein bioreceptors like antibodies, which can easily be adsorbed on gold surfaces due to the high content of amine groups, but their ability to capture and detect antigens essentially depends on their end-on orientation (with Fab regions available for antigen binding) and correct secondary structural conformation. A similar and better possibility is chemisorption. Thiol groups (-SH) are known to strongly interact with gold or silver, losing the hydrogen atom and forming a chemical bond. Therefore, bioreceptors with available thiol groups can be directly chemisorbed on the sensor surface, as can be done, for example, with antibody fragments, aptamers, or DNA probes. It is important to take into account, though, that lateral spacer molecules, such as short chain alkanethiols, might be used to control the bioreceptor density and reduce possible steric hindrance for analyte capture.

Another widely used and convenient strategy for bioreceptor immobilization is the covalent binding to a functional chemical matrix formed on the sensor surface [Fig. 7(b)]. For plasmonic sensors, the two most common scaffolds are dextran-based polymers and alkanethiol self-assembled monolayers (SAMs). In both cases, a carboxyl $(\mathrm{COOH})$ functional group is usually incorporated into the chemical scaffold so that it can be activated for binding to terminal amine $\left(\mathrm{NH}_{2}\right)$ groups, largely available in protein bioreceptors. This chemical reaction involves a well-established procedure employing a carbodiimide-based chemistry (i.e., EDC/NHS) and results in very stable amide bonds between the protein and the functionalized sensor surface. Other covalent coupling chemistries can also be used, for example, amine-amine crosslinking or click chemistry, among others. This covalent binding methodology offers important advantages, such as the high control of bioreceptor density, by adjusting the number of functional groups or by including lateral spacers, and it also provides a good coverage of the metal surface for preventing non-specific adsorptions. The antifouling properties of the SAMs can be further enhanced by using PEGylated molecules, which, due to their high hydrophilicity, provide protein adsorption resistance. However, the direct covalent binding of bioreceptors generally occurs randomly, without any preferred orientation. To ensure a unidirectional immobilization of bioreceptors, it is recommended to make use of affinity ligands. For example, protein $A / G$ are known to present a high affinity for the constant region of antibodies $(\mathrm{Fc})$, therefore allowing its immobilization in the correct end-on orientation [Fig. 7(c)]. Another widely used strategy is the use of the biotin/streptavidin pair. Antibodies can be biotinylated specifically through the carbohydrate moieties of the $\mathrm{Fc}$ region and subsequently attach to a streptavidin layer formed on the sensor surface [Fig. 7(d)]. In addition to these methods, a vast number of different bioengineering strategies have been proposed for sensor functionalization, e.g., histidine or cysteine tags, calixarenes [Fig. 7(e)], DNA-mediated coupling [Fig. 7(f)], etc., for which we refer to specific articles and specialized reviews. ${ }^{7-74}$

Another important aspect in biofunctionalization is related to the surface regeneration, that is, the removal of the target analyte after the detection step without altering the immobilized bioreceptor 
layer. Efficient regeneration would provide reusability of the sensor, which is particularly important not only to save costs and time, but also to evaluate the stability and robustness of the bioactive surface. Typically, regeneration can be accomplished by introducing a low or high $\mathrm{pH}$ solution (e.g., $\mathrm{HCl}, \mathrm{NaOH}$, glycine, etc.) that disrupts the biochemical interaction between the analyte and the receptor. Other methods make use of ionic strength changes (e.g., high/low salt content buffers), temperature, or specific chemicals (e.g., formamide for DNA dehybridization) to break the interaction. Nevertheless, the regeneration procedures have to be evaluated empirically since the combination of binding forces is often unknown, and it is important to select the mildest regeneration conditions that assure the stability and integrity of the biorecognition layer.

Finally, one of the critical factors in biosensor functionalization is related to the selectivity and antifouling properties of the biolayer. Particularly for label-free plasmonic sensors that detect changes in RI (and, therefore, the changes of mass on the surface), the non-specific adsorption of sample matrix components is a major challenge for real applications. A number of strategies have been employed to reduce the adsorption of non-relevant components: the use of PEGylated polymers as functional layers, the addition of blocking proteins like albumins or PEGylated polymers to saturate the surface, or a combination of both. However, the effect of these approaches is not fully controlled, and they are generally combined with sample dilution or buffer additives to minimize the background signal of real samples. In this regard, nanoplasmonic biosensors based on nanoparticle arrays might possess an interesting advantage compared with homogeneous thin film SPR biosensors: the site-selective biofunctionalization. By exploiting the different reactivities of two different materials (e.g., gold and glass), it is possible to immobilize the bioreceptors specifically onto the plasmonic particles and block the exposed surface of the substrate to assure its inertness. In such a way, all relevant biomolecular interactions will occur solely within the sensing probe areas and non-specific binding can be effectively minimized, which will increase the overall sensitivity and selectivity, thus improving the total bioassay performance.

\section{APPLICATIONS OF PLASMONIC BIOSENSORS}

Plasmonic biosensing technology is one of the most versatile analytical techniques available nowadays. By choosing the appropriate biorecognition element, it can be applied to virtually any type of target molecule, including proteins and nucleic acids, but also small molecules like drugs or contaminants, pathogens like viruses or bacteria, and even human cells. It is so that a myriad of works have been reported demonstrating their application, especially in the biomedical and environmental fields. Rather than mentioning about and commenting upon several examples in the literature, for which we refer to excellent published reviews, ${ }^{4,75,76}$ in this tutorial, we will use a relevant case to illustrate and discuss the general procedure to be followed when designing and validating plasmonic biosensor applications for a specific purpose.

In medicine, the accurate diagnosis of specific diseases is crucial for the timely administration of correct treatment and appropriate clinical management of the patient. Further, the rapid and early identification of certain diseases, before the appearance of external symptomatology, can be extremely important. This is the case of cancer, for example, which has been shown to have much better prognosis and patient survival rates when it is detected and treated at early stages. ${ }^{77}$ Another clear case has been seen with the COVID-19 pandemic in 2020. This infectious disease emerged in late 2019 in Wuhan (China), caused by a coronavirus named SARS-CoV-2 (severe acute response syndrome coronavirus 2). Due to the fast and easy transmission of the virus (i.e., airborne transmission), the disease has rapidly spread worldwide, with pretty much no other options to control and stop it than population lockdown and social distancing. The availability of plasmonic biosensors for rapid and sensitive detection of SARS-CoV-2 viruses might be significantly useful for massive population screening, early detection of infected patients, and a more efficient management of the pandemic. ${ }^{78}$

To develop a biosensor for an emergent virus diagnosis, the first steps to be taken into account are the bioassay approach and the specific bioreceptors, which will be based on the viral target molecule. Ideally, the preferred approach might be to target the external viral antigens, such as the spike (S) proteins of the coronavirus. This will allow direct detection and quantification of the number of viruses in a patient. For this, it is required to produce de novo antibodies with sufficient affinity and selectivity toward the protein. This procedure can be relatively long and complex, since it needs to fully characterize the viral antigen, produce it (e.g., bacterial-based recombinant proteins), and then obtain the antibodies either by animal immunization and subsequent purification or by recombinant methods, for example. Once an appropriate bioreceptor is achieved, the sensor surface biofunctionalization has to be designed and evaluated, controlling the orientation, density, and distribution of the specific antibodies to enhance the virus capture and detection. It is especially important to consider the viral particle sizes and mass transport issues, plus the physicochemical assay conditions, such as flow rates, $\mathrm{pH}$, buffer composition, etc. In parallel, the target clinical sample has to be rationally selected and established. It has been demonstrated that COVID-19 viruses are mostly present in respiratory fluids (nasal and nasopharyngeal) as well as in saliva. The pros and cons can be listed for the two types of clinical specimens. Respiratory fluids are collected with specific swabs, which might be a little disturbing for patients (especially for children) but, when performed by trained sanitary personnel, it ensures reliable virus sampling and storage. Saliva collection, in contrast, is non-invasive and can be easily done by the patient itself. However, saliva sampling may present a much higher variability, depending on the collection time and method, which can lead to assay reproducibility and reliability issues. Either way, both the sensor biofunctionalization and bioassay conditions will be assessed and finely optimized in terms of sample matrix effects (background signal and antibody-antigen recognition), to ensure the optimal analytical performance. Standard calibration curves will be obtained by spiking buffer and biological samples with known concentrations of the target (e.g., viruses obtained in microbiological culture), which will allow determining the analytical parameters, such as the concentration sensitivity, limit of detection and quantification, assay reproducibility and coefficients of variation, etc. Finally, the biosensor must be validated through the analysis of a number of blind clinical samples. It is recommended to 
challenge the novel biosensor vs standard techniques (e.g., ELISA, PCR), if available. With a large validation study $(\mathrm{n}>100)$, the clinical sensitivity and specificity may be determined, besides the accuracy, positive and negative predicted values, etc.

Owing to the versatility of plasmonic biosensors, it is also feasible to develop COVID-19 diagnostics based on other analytical approaches. For instance, it will be possible to target the genomic RNA sequence of the virus, instead of the viral antigens. Thanks to the advanced next-generation sequencing (NGS), in just a few weeks' time, the exact genome of the SARS-CoV-2 virus was deciphered. This enabled the rapid development of highly specific genomic assays, based on RT-PCR, which have become the gold standard in COVID-19 diagnosis. Plasmonic biosensors can be applied for the direct and label-free detection of viral RNA by designing and immobilizing on the sensor surface single-stranded DNA probes with complementary sequence to specific SARS-CoV-2 gene fragments. Compared with the antigen-based recognition assay, genomic analysis is advantageous in regard to the bioreceptor, which can be custom-designed and synthetically produced, but it requires the sample to be treated and processed for RNA extraction and fragmentation, and an outstanding sensor sensitivity will be needed when attempting direct RNA detection without previous PCR amplification. To further enhance the diagnostic capabilities, multiplexed plasmonic sensor systems could be implemented. The sensitivity and specificity of SARS-CoV-2 virus could be increased by combining different DNA probes targeting distinct genes of the same virus, but it might also be possible to realize one platform able to differentiate among several viruses sharing clinical symptomatology with COVID-19, e.g., human coronaviruses (hCoVs) responsible for common colds, or influenza viruses responsible for flu.

On these lines, the same plasmonic biosensor technology could also be employed for environmental control and monitoring. The zoonotic origin of the COVID-19 pandemic has evidenced the need for accurately studying the presence and evolution of viruses in animals, especially for those colonies that share spaces with humans or are susceptible of being in close contact, eaten, or treated in some way. Similar cases occurred with the Ebola virus and the bird or swine flu, or more commonly with bacterial infections, like salmonellosis. Simple, automated, and portable plasmonic sensors could enable a rapid detection of risky pathogens in animals, food, or water, and prevent from human transmission and pandemic outbreaks. For the particular case of COVID-19, it is known that bats and certain rodents are the main reservoirs of coronaviruses. Among them, one should distinguish the different families, since alphacoronaviruses are mostly harmless for humans, while betacoronaviruses are prone to cause severe diseases, such as the SARS-CoV in 2003, the MERS-CoV in 2012, or the current SARS-CoV-2. Plasmonic biosensors targeting specific regions could rapidly and reliably detect the presence of these dangerous viruses. Furthermore, if integrated in point-of-care systems, plasmonic biosensors could be employed directly on field to carry out a close and routine monitoring of animal reservoir colonies, which would aid in early warning of communities and authorities, so they can urgently take the corresponding actions to minimize the risk of new pandemics.

Finally, it must be mentioned that developing applications for plasmonic biosensors should focus not only on the biochemical and analytical aspects, but also on the integration of microfluidic systems for sample handling as well as data analysis and interpretation. The engineering of microfluidics is continuously evolving, incorporating innovative designs and materials that can greatly facilitate and improve the biosensor performance. The goal of accomplishing a handy lab-on-a-chip system that includes sample processing and treatment, accurate and reliable analysis, and waste disposal management is still the most ambitious one in biosensor research. In parallel, the speedy development of artificial intelligence and machine learning tools will also effectively impact biosensor applications. The implementation of advanced algorithms for signal processing and interpretation could signify a breakthrough in biosensors, which would become smart systems not limited to detecting and quantifying specific analytes but also aiding in the decision-making process, even directly connected to therapy delivery systems or data management clouds.

\section{SUMMARY AND FUTURE PERSPECTIVES}

Plasmonic materials and nanostructures have a unique and vastly demonstrated potential for the realization of new enabling biosensor technologies with exceptional analytical capabilities. Plasmonic biosensors, through the evanescent wave based working principle, can offer label-free detection of any type of analyte (i.e., proteins, nucleic acids, pathogens, drugs, etc.) with outstanding sensitivities and in a real-time one-step format. Furthermore, they can be integrated in small footprint, portable, and user-friendly devices to be employed directly at the point of need, which is key for their application in massive clinical analyses or for on-field environmental monitoring. For the design and development of plasmonic and nanoplasmonic biosensors, one may take into account several factors, including the selection of the plasmonic metal, the particle geometry, the optical configuration and instrumentation, and also the biorecognition interface, which must be carefully optimized for each specific application. When all aspects are considered and finely engineered, plasmonic biosensor performance can meet the requirements for addressing relevant bioanalytical goals, such as the early diagnosis of serious diseases like cancer, massive population screening for virus infection detection, or for routine testing of contaminants in food or water.

In terms of biosensor performance, on-going research is already looking into new materials (e.g., high refractive index semiconductors) and sophisticated architectures that can provide the narrowest resonance bandwidth and largest refractometric sensitivity, as well as innovative nanofabrication methods and substrate supports that enable the development of flexible and/or wearable sensors. The introduction of novel bioengineered receptors and 2D materials as chemical biofunctionalization scaffolds might also enhance the analytical sensitivity and specificity of label-free plasmonic biosensors. Regarding device instrumentation, in the near future, plasmonic biosensors could become fully automated and smart miniaturized devices, integrated as powerful lab-on-a-chip tools. For this purpose, research in photonics engineering should synergize with other areas like materials and computer sciences, to implement cutting-edge technologies in microfluidics and data treatment that assure maximum accuracy and reliability of the 
biosensor devices as well as their regulatory approval and acceptance in clinics and environmental analysis.

\section{ACKNOWLEDGMENTS}

Financial support is acknowledged from the SensCELL project (Ref. No. PGC-2018-099870), funded by the Spanish Ministry of Science and Innovation, the Spanish Research Agency (AEI), and the European Regional Development Fund (ERDF). The ICN2 is funded by the CERCA programme/Generalitat de Catalunya. The ICN2 is supported by the Severo Ochoa Centres of Excellence programme, funded by AEI (Grant No. SEV-2017-0706).

\section{DATA AVAILABILITY}

Data sharing is not applicable to this article as no new data were created or analyzed in this study.

\section{REFERENCES}

${ }^{1}$ B. Heidt, W. F. Siqueira, K. Eersels, H. Diliën, B. Van Grinsven, R. T. Fujiwara, and T. J. Cleij, "Point of care diagnostics in resource-limited settings: A review of the present and future of $\mathrm{PoC}$ in its most needed environment," Biosensors 10(10), 133 (2020).

${ }^{2}$ A. Romeo, T. S. Leung, and S. Sánchez, "Smart biosensors for multiplexed and fully integrated point-of-care diagnostics,” Lab Chip 16(11), 1957-1961 (2016).

${ }^{3}$ N. Kalyani, S. Goel, and S. Jaiswal, "On-site sensing of pesticides using point-of-care biosensors: A review,” Environ. Chem. Lett. 1, 3 (2020).

${ }^{\mathbf{4}}$ M. Soler, C. S. Huertas, and L. M. Lechuga, "Label-free plasmonic biosensors for point-of-care diagnostics: A review," Expert Rev. Mol. Diagn. 19(1), 71-81 (2019).

${ }^{\mathbf{5}}$ F. S. Ligler and J. J. Gooding, "Lighting up biosensors: Now and the decade to come," Anal. Chem. 91(74), 8732-8738 (2019).

${ }^{6}$ D. Liu, J. Wang, L. Wu, Y. Huang, Y. Zhang, M. Zhu, Y. Wang, Z. Zhu, and C. Yang, "Trends in miniaturized biosensors for point-of-care testing," TrAC 122, 115701 (2020).

${ }^{7}$ R. T. Hill, "Plasmonic biosensors," Wiley Interdiscip. Rev. Nanomed. Nanobiotechnol. 7(2), 152-168 (2015).

${ }^{8}$ R. W. Wood, "On a remarkable case of uneven distribution of light in a diffraction grating spectrum,” Proc. Phys. Soc. London 18(1), 269-275 (1901).

${ }^{9}$ R. W. Wood, "XXVII. Diffraction gratings with controlled groove form and abnormal distribution of intensity," London, Edinburgh, Dublin Philos. Mag. J. Sci. 23(134), 310-317 (1912).

${ }^{10} \mathrm{~L}$. Rayleigh and L. Rayleigh, "On the dynamical theory of gratings," Proc. R. Soc. London. Ser. A Contain. Pap. Math. Phys. Character. 79(532), 399-416 (1907).

${ }^{11}$ R. W. Wood, “Anomalous diffraction gratings," Phys. Rev. 48(12), 928-936 (1935).

${ }^{12}$ C. H. Palmer, "Parallel diffraction grating anomalies*," J. Opt. Soc. Am. 42(4), 269 (1952).

${ }^{13}$ C. H. Palmer, "Diffraction grating anomalies II coarse gratings ${ }^{\star}$," J. Opt. Soc. Am. 46(1), 50 (1956).

${ }^{14} \mathrm{U}$. Fano, "The theory of anomalous diffraction gratings and of quasi-stationary waves on metallic surfaces (Sommerfeld's waves)," J. Opt. Soc. Am. 31(3), 213 (1941).

${ }^{15} \mathrm{D}$. Bohm and D. A. Pines, "Collective description of electron interactions. I. Magnetic interactions,” Phys. Rev. 82(5), 625-634 (1951).

${ }^{16}$ D. Pines and D. A. Bohm, "Collective description of electron interactions: II. Collective vs individual particle aspects of the interactions," Phys. Rev. 85(2), 338-353 (1952).

${ }^{17}$ D. A. Pines, "Collective description of electron interactions: IV. Electron interaction in metals," Phys. Rev. 92(3), 626-636 (1953).
${ }^{18}$ E. Kretschmann and H. Raether, "Radiative decay of non radiative surface plasmons excited by light," Phys. Rev. Lett. 19, 398 (1968).

${ }^{19}$ A. Otto, "Excitation of nonradiative surface plasma waves in silver by the method of frustrated total reflection," Z. Phys. 216(4), 398-410 (1968).

${ }^{\mathbf{2 0}} \mathrm{B}$. Liedberg, C. Nylander, and I. Lunström, "Surface plasmon resonance for gas detection and biosensing," Sensors Actuators 4(C), 299-304 (1983).

${ }^{21}$ D. C. Cullen, R. G. W. Brown, and C. R. Lowe, "Detection of immunocomplex formation via surface plasmon resonance on gold-coated diffraction gratings," Biosensors 3(4), 211-225 (1987).

${ }^{22}$ I. Pockrand, J. D. Swalen, J. G. Gordon, and M. R. Philpott, "Surface plasmon spectroscopy of organic monolayer assemblies," Surf. Sci. 74(1), 237-244 (1978).

${ }^{23}$ M. Malmqvist, "BIACORE: An affinity biosensor system for characterization of biomolecular interactions," Biochem. Soc. Trans. 27, 335-340 (1999).

${ }^{\mathbf{2 4}}$ B. Liedberg, C. Nylander, and I. Lundström, "Biosensing with surface plasmon resonance-How it all started," Biosens. Bioelectron. 10(8), i-ix (1995).

${ }^{\mathbf{2 5}}$ J. J. Burke, G. I. Stegeman, and T. Tamir, "Surface-polariton-like waves guided by thin,” Lossy Metal Films. Phys. Rev. B 33(8), 5186-5201 (1986).

${ }^{\mathbf{2 6}}$ D. R. Tilley, "Surface polaritons: Electromagnetic waves at surfaces and interfaces,” Opt. Acta Int. J. Opt. 30(11), 1501-1501 (1983).

27. Homola, in Optical Biosensors, 2nd ed., edited by F. S. Ligler and C. Rowe Taitt (Elsevier, 2008), pp. 185-242.

${ }^{28}$ Y. Gutiérrez, A. S. Brown, F. Moreno, and M. Losurdo, "Plasmonics beyond noble metals: Exploiting phase and compositional changes for manipulating plasmonic performance,” J. Appl. Phys. 128(8), 080901 (2020).

${ }^{29}$ Y. Yang, I. I. Kravchenko, D. P. Briggs, and J. Valentine, "All-dielectric metasurface analogue of electromagnetically induced transparency," Nat. Commun. 5(1), 5753 (2014)

${ }^{30}$ A. N. Grigorenko, M. Polini, and K. S. Novoselov, "Graphene plasmonics," Nat. Photonics 6(11), 749-758 (2012).

${ }^{31}$ J. Homola and M. Piliarik, "Surface plasmon resonance (SPR) sensors," in Springer Series on Chemical Sensors and Biosensors, 4th ed. (Springer, 2006), pp. 45-67.

${ }^{32}$ B. Prabowo, A. Purwidyantri, and K.-C. Liu, "Surface plasmon resonance optical sensor: A review on light source technology," Biosensors 8(3), 80 (2018).

${ }^{33}$ J. Dostálek, J. Čtyroký, J. Homola, E. Brynda, M. Skalský, P. Nekvindová, J. Špirková, J. Škvor, and J. Schröfel, "Surface plasmon resonance biosensor based on integrated optical waveguide," Sens. Actuators B 76, 8-12 (2001).

${ }^{34}$ A. A. Rifat, R. Ahmed, A. K. Yetisen, H. Butt, A. Sabouri, G. A. Mahdiraji, S. H. Yun, and F. R. M. Adikan, "Photonic crystal fiber based plasmonic sensors," Sens. Actuators B 243, 311-325 (2017).

${ }^{35}$ A. K. Sharma, R. Jha, and B. D. Gupta, "Fiber-optic sensors based on surface plasmon resonance: A comprehensive review," IEEE Sensors J. 7(8), 1118-1129 (2007).

${ }^{36}$ C. Caucheteur, T. Guo, and J. Albert, "Polarization-Assisted fiber bragg grating sensors: Tutorial and review," J. Light. Technol. 35(16), 3311-3322 (2017).

37J. Homola, S. S. Yee, and G. Gauglitz, "Surface plasmon resonance sensors: Review,” Sensors Actuators B Chem. 54(1), 3-15 (1999).

${ }^{38} \mathrm{C}$. L. Wong and M. Olivo, "Surface plasmon resonance imaging sensors: A review," Plasmonics 9(4), 809-824 (2014).

${ }^{39} \mathrm{~J}$. Homola, "On the sensitivity of surface plasmon resonance sensors with spectral interrogation," Sensors Actuators B Chem. 41(1-3), 207-211 (1997).

${ }^{40}$ W.-C. Kuo, C. Chou, and H.-T. Wu, "Optical heterodyne surface-plasmon resonance biosensor,” Opt. Lett. 28(15), 1329 (2003).

${ }^{41}$ S. Deng, P. Wang, and X. Yu, "Phase-sensitive surface plasmon resonance sensors: Recent progress and future prospects," Sensors 17(12), 2819 (2017).

${ }^{42}$ Z. Geng, X. Zhang, Z. Fan, X. Lv, Y. Su, and H. Chen, "Recent progress in optical biosensors based on smartphone platforms," Sensors 17(11), 2449 (2017).

${ }^{43}$ H. Guner, E. Ozgur, G. Kokturk, M. Celik, E. Esen, A. E. Topal, S. Ayas, Y. Uludag, C. Elbuken, and A. A. Dana, "Smartphone based surface plasmon resonance imaging (SPRi) platform for on-site biodetection," Sensors Actuators B Chem. 239, 571-577 (2017). 
${ }^{44}$ P. Preechaburana, M. C. Gonzalez, A. Suska, and D. Filippini, "Surface plasmon resonance chemical sensing on cell phones," Angew. Chem. Int. Ed. 51(46), 11585-11588 (2012).

${ }^{45}$ I. Freestone, N. Meeks, M. Sax, and C. Higgitt, "The Lycurgus Cup-A Roman nanotechnology,” Gold Bull. 40(4), 270-277 (2008).

${ }^{46} \mathrm{~K}$. M. Mayer and J. H. Hafner, "Localized surface plasmon resonance sensors," Chem. Rev. Am. Chem. Soc. 111, 3828-3857 (2011).

${ }^{47}$ J. Zhao, X. Zhang, C. R. Yonzon, A. J. Hoes, and R. P. Van Duyne, "Localized surface plasmon resonance biosensors," Nanomedicine 1(2), 219-228 (2006).

${ }^{48} \mathrm{E}$. Hutter and J. H. Fendler, "Exploitation of localized surface plasmon resonance," Adv. Mater. 16(19), 1685-1706 (2004).

${ }^{49}$ M. A. Otte, B. Sepúlveda, W. Ni, J. P. Juste, L. M. Liz-Marzán, and L. M. Lechuga, "Identification of the optimal spectral region for plasmonic and nanoplasmonic sensing," ACS Nano 4(1), 349-357 (2010).

${ }^{50}$ M. F. Limonov, M. V. Rybin, A. N. Poddubny, and Y. S. Kivshar, "Fano resonances in photonics," Nat. Photonics 11(9), 543-554 (2017).

${ }^{51}$ M. R. Jones, K. D. Osberg, R. J. MacFarlane, M. R. Langille, and C. A. Mirkin, "Templated techniques for the synthesis and assembly of plasmonic nanostructures," Chem. Rev. 111(6), 3736-3827 (2011)

${ }^{52} \mathrm{Y}$. Yin and A. P. Alivisatos, "Colloidal nanocrystal synthesis and the organic-inorganic interface," Nature 437(7059), 664-670 (2005).

${ }^{53} \mathrm{~K}$. Du, J. Ding, Y. Liu, I. Wathuthanthri, and C.-H. Choi, "Stencil lithography for scalable micro- and nanomanufacturing," Micromachines 8(4), 131 (2017).

${ }^{54}$ Q. Xie, M. H. Hong, H. L. Tan, G. X. Chen, L. P. Shi, and T. C. Chong, "Fabrication of nanostructures with laser interference lithography," J. Alloys Compounds 449(1-2), 261-264 (2008).

${ }^{55} \mathrm{~K}$. Xiong, G. Emilsson, and A. B. Dahlin, "Biosensing using plasmonic nanohole arrays with small, homogenous and tunable aperture diameters," Analyst 141(12), 3803-3810 (2016).

${ }^{56} \mathrm{C}$. Escobedo, "On-chip nanohole array based sensing: A review," Lab Chip 13(13), 2445 (2013).

${ }^{57}$ A. Prasad, J. Choi, Z. Jia, S. Park, and M. R. Gartia, "Nanohole array plasmonic biosensors: Emerging point-of-care applications," Biosensors Bioelectronics 130, 185-203 (2019).

${ }^{58} \mathrm{H}$. Chen, X. Kou, Z. Yang, W. Ni, and J. Wang, "Shape- and size-dependent refractive index sensitivity of gold nanoparticles," Langmuir 24(10), 5233-5237 (2008).

${ }^{59}$ H. Chen, L. Shao, K. C. Woo, T. Ming, H. Q. Lin, and J. Wang, "Shape-dependent refractive index sensitivities of gold nanocrystals with the same plasmon resonance wavelength," J. Phys. Chem. C 113(41), 17691-17697 (2009).

${ }^{60} \mathrm{G}$. K. Joshi, P. J. McClory, S. Dolai, and R. Sardar, "Improved localized surface plasmon resonance biosensing sensitivity based on chemically-synthesized gold nanoprisms as plasmonic transducers," J. Mater. Chem. 22(3), 923-931 (2012).

${ }^{61}$ A. E. Cetin, S. Kaya, A. Mertiri, E. Aslan, S. Erramilli, H. Altug, and M. Turkmen, "Dual-band plasmonic resonator based on jerusalem cross-shaped nanoapertures," Photonics Nanostruct. Fundam. Appl. 15, 73-80 (2015).

${ }^{62}$ N. Verellen, P. Van Dorpe, C. Huang, K. Lodewijks, G. A. E. Vandenbosch, L. Lagae, and V. V. Moshchalkov, "Plasmon line shaping using nanocrosses for high sensitivity localized surface plasmon resonance sensing," Nano Lett. 11(2), 391-397 (2011)
${ }^{63}$ J. Homola, I. Koudela, and S. S. Yee, "Surface plasmon resonance sensors based on diffraction gratings and prism couplers: Sensitivity comparison," Sensors Actuators B Chem. 54(1), 16-24 (1999).

${ }^{64}$ M. Soler, M.-C. Estevez, M. Alvarez, M. A. Otte, B. Sepulveda, and L. M. Lechuga, "Direct detection of protein biomarkers in human fluids using site-specific antibody immobilization strategies," Sensors 14(2), 2239 (2014).

${ }^{65} \mathrm{P}$. Offermans, M. C. Schaafsma, S. R. K. Rodriguez, Y. Zhang, M. Crego-Calama, S. H. Brongersma, and J. Gómez Rivas, "Universal scaling of the figure of merit of plasmonic sensors," ACS Nano 5(6), 5151-5157 (2011).

${ }^{66}$ A. A. Yanik, A. E. Cetin, M. Huang, A. Artar, S. H. Mousavi, A. Khanikaev, J. H. Connor, G. Shvets, and H. Altug, "Seeing protein monolayers with naked eye through plasmonic Fano resonances," Proc. Natl. Acad. Sci. U.S.A. 108(29), 11784-11789 (2011)

${ }^{67}$ S. Zhang, K. Bao, N. J. Halas, H. Xu, and P. Nordlander, "Substrate-induced Fano resonances of a plasmonic nanocube: A route to increased-sensitivity localized surface plasmon resonance sensors revealed," Nano Lett. 11(4), 1657-1663 (2011)

${ }^{68}$ T. G. Habteyes, S. Dhuey, E. Wood, D. Gargas, S. Cabrini, P. J. Schuck, A. P. Alivisatos, and S. R. Leone, "Metallic adhesion layer induced plasmon damping and molecular linker as a nondamping alternative," ACS Nano 6(6), 5702-5709 (2012).

${ }^{69}$ B. Brian, B. Sepúlveda, Y. Alaverdyan, L. M. Lechuga, and M. Käll, "Sensitivity enhancement of nanoplasmonic sensors in low refractive index substrates," Opt. Express 17(3), 2015 (2009)

${ }^{70}$ M. A. Otte, M. C. Estévez, L. G. Carrascosa, A. B. González-Guerrero, L. M. Lechuga, and B. Sepúlveda, "Improved biosensing capability with novel suspended nanodisks," J. Phys. Chem. C 115(13), 5344-5351 (2011).

${ }^{71}$ M. Oliverio, S. Perotto, G. C. Messina, L. Lovato, and F. De Angelis, "Chemical functionalization of plasmonic surface biosensors: A tutorial review on issues, strategies, and costs," ACS Appl. Mater. Interfaces 9(35), 29394-29411 (2017).

${ }^{72}$ E. Mauriz, M. C. García-Fernández, and L. M. Lechuga, "Towards the design of universal immunosurfaces for SPR-based assays: A review," TrAC 79, 191-198 (2016).

${ }^{73}$ M. Soler, C. S. Huertas, and L. M. Lechuga, "Label-free plasmonic biosensors for point-of-care diagnostics: A review,” Expert Rev. Mol. Diagn. 19, 71-81 (2019).

${ }^{74}$ C. S. Huertas, M. Soler, M.-C. Estevez, and L. M. Lechuga, “One-Step immobilization of antibodies and DNA on gold sensor surfaces via a poly-adenine oligonucleotide approach,” Anal. Chem. 92(18), 12596 (2020).

${ }^{75}$ Y. Saylan, S. Akgönüllü, and A. Denizli, "Plasmonic sensors for monitoring biological and chemical threat agents," Biosensors 10(10), 142 (2020).

${ }^{76}$ G. Zanchetta, R. Lanfranco, F. Giavazzi, T. Bellini, and M. Buscaglia, "Emerging applications of label-free optical biosensors," Nanophotonics 6(4), 627-645 (2017).

${ }^{77}$ N. Bellassai, R. D’Agata, V. Jungbluth, and G. Spoto, "Surface plasmon resonance for biomarker detection: Advances in non-invasive cancer diagnosis," Front. Chem. 7, 570 (2019).

${ }^{78}$ M. Soler, M. C. Estevez, M. Cardenosa-Rubio, A. Astua, and L. M. Lechuga, "How nanophotonic label-free biosensors can contribute to rapid and massive diagnostics of respiratory virus infections: COVID-19 case," ACS Sensors 5(9), 2663-2678 (2020). 